\title{
Soft X-ray analysis of a loop flare on the Sun
}

\author{
J. I. Khan ${ }^{1, \star}$, H. S. Hudson ${ }^{2}$, and Z. Mouradian ${ }^{3}$ \\ 1 Tuorla Observatory ${ }^{\star \star}$, University of Turku, Väisäläntie 20, 21500 Piikkiö, Finland \\ 2 Space Sciences Laboratory, University of California, Berkeley, Centennial Drive, CA 94720-7450, USA \\ 3 Observatoire de Paris, Section de Meudon, LESIA, 5 place Jules Janssen, 92195 Meudon Cedex, France
}

Received 3 September 2003 / Accepted 26 November 2003

\begin{abstract}
We present the results of an analysis of soft X-ray images for a solar flare which occurred on 1992 July 11. This flare, as seen in Yohkoh Soft X-ray Telescope (SXT) images was of comparatively simple geometry, consisting of two bright footpoints early in the flare with a bright loop seen later in the flare. We examine how closely this flare compares with the supposed paradigm of a confined simple-loop flare. Closer examination of the SXT images reveals that the flare structure consisted of at least two adjacent loops, one much fainter than the other. We examine the brighter of the two soft X-ray loops. The SXT images reveal an apparent slow, northward motion of this loop (roughly transverse to its major axis). Examination of derived emission measure and temperature images also indicate an apparent northward motion. In addition, we find an increase in the cross-sectional width at the top of the loop with time. Emission measure maps derived from the SXT images also indicates an apparent broadening of the loop-top region. We infer that the apparent northward motion and the apparent broadening of the soft X-ray emission can be explained in a reconnection scenario where successive magnetic field structures do not lie in a plane but are tilted to the south of the line of sight but with successively brightening loops oriented at less tilted angles. H $\alpha$ images for this flare reveal an evolution from a few brilliant points to a short two-ribbon-like appearance. Comparison of the SXT images with the $\mathrm{H} \alpha$ images shows that the $\mathrm{H} \alpha$ patches are aligned with the footpoints of the soft X-ray loops, suggesting the presence of a small arcade structure. There is no clear evidence for an eruptive signature in our observations nor in reports from other observations. The lack of an eruptive signature could suggest that the flare may have been a confined simple-loop flare, but this is not compelling due to a gap in the coronal observations prior to and early in the event. Analysis of our observations indicate that the flare exhibited characteristics suggesting that it may be better understood as a mini-arcade flare. These results casts doubt on the validity of the supposed paradigm of a confined simple-loop flare, at least for this flare. They indicate that even an apparently simple-loop flare may be considered to be a variety of arcade flare. We also find an effect which, to our knowledge, has not been reported before: the hot flaring regions later become cooler than the surrounding quiescent corona. That is, the flare loops do not evolve into bright active region loops, but into cooler loops. This may indicate an increase in the efficiency of the cooling mechanism or a transformed equilibrium state within the flaring loops.
\end{abstract}

Key words. Sun: corona - Sun: flares - Sun: X-rays, gamma rays

\section{Introduction}

It has long been known that differences exist between solar flares, such as differences in scale size, duration, as well as qualitative and quantitative differences in their emissions. An important issue in the understanding of solar flares is whether these, or other differences, are significant and justify a categorization scheme for flares into different types. Such a categorization, if valid, may imply the existence of different physical mechanisms for the different flare types, or indicate the different physical conditions in which flares may occur.

Concerning the question of flare categorizations, Pallavicini et al. (1977), based on an analysis of images

Send offprint requests to: J. I. Khan, e-mail: jkhan@spd.aas.org

* Current address: Department of Physics and Astronomy, University of Glasgow, Glasgow G12 8QQ, Scotland, UK.

$\star \star$ Tuorla Observatory is part of the Väisälä Institute for Space Physics and Astronomy (VISPA), University of Turku. of limb flares obtained from the S-054 grazing incidence soft X-ray telescope (Vaiana et al. 1977) on board Skylab, suggested that solar flares could be classified into two broadly different categories: short-duration compact flares and long-duration large-scale flares, with physically distinct characteristics. Subsequent examination of the S-054 data, as well as an analysis of data from the Flat Crystal Spectrometer of the X-ray Polychromator experiment (Acton et al. 1980; Culhane et al. 1984) on the Solar Maximum Mission (Bohlin et al. 1980) led Pallavicini (1982) to reach broadly similar conclusions on the subject of flare categorizations. However, in a later reassessment of these data and results Pallavicini (1991) retreated from such categorizations. Despite this, the concepts of compact and large-scale flares and that there are significant differences between them, persists.

Generally speaking the two flare categories have been based on differences in size scales, durations and particle 
densities, and on the inferred need to explain these and other differences. Large-scale flares have also been referred to as long-duration events (LDEs), eruptive, ejective, arcade or tworibbon flares, while compact flares have also been referred to as confined, impulsive or simple-loop flares. Henceforth, we use the terms arcade flare and simple-loop flare to refer to these two categories of flares.

Simple-loop flares have been categorized as being small in size, high in particle number density, and short in duration, while arcade have been categorized as being large in size, lower in particle number density and longer in duration. Simple-loop flares have been associated with one or a few hard X-ray bursts, while arcade flares may have many hard X-ray bursts. In soft X-ray images simple-loop flares appear to consist of one or perhaps a few compact coronal loops, while arcade flares show a clear arcade of loops. Arcade flares have usually been associated with the eruption of an active region filament or quiescent filament often seen in $\mathrm{H} \alpha$, while no such association has generally been presumed for simple-loop flares. In addition, coronal mass ejections tend to be more closely associated with long-duration soft X-ray (arcade) flares than with shorter-lived (compact) flares (Kahler et al. 1989; Kay et al. 2003).

The energy release for a simple-loop flare is sometimes ascribed to changes in the magnetic field configuration internal to the magnetic loop(s) but without changes to larger-scale magnetic field structures (e.g., magnetic reconnection via tearing modes inside a twisted magnetic loop, Spicer 1977). An alternative suggestion is the emerging flux model (Heyvaerts et al. 1977; Nishio et al. 1997) where an emerging small-scale loop interacts with overlying larger-scale magnetic structures and the bulk of the energy is released within the loop. In the emerging flux model if there are open or extended magnetic field lines present then some particles may escape giving rise to type III radio bursts, remote microwave emission, and mass ejections in the form of X-ray jets or surges seen in $\mathrm{H} \alpha$ or at other wavelengths. Thus, for the emerging flux model the flare is, strictly speaking, not entirely confined. Flare loop models have also been proposed which involve interactions between two twisted coronal loops (e.g., Gold \& Hoyle 1960; Melrose 1997; Sakai $\&$ de Jager 1996).

Pearce \& Harrison (1988) and Yeung \& Pearce (1990), based on the statistical analyses of a few simple flare parameters for a large number of flares, argued that there was no evidence supporting the hypothesis of different types of flares. However, recently Temmer et al. (2001) and Veronig et al. (2002) have shown that some differences in flare classes do indeed exist when the statistical data are considered more appropriately.

Examination of images from the Soft X-ray Telescope (SXT) (Tsuneta et al. 1991) on board the Yohkoh satellite (Ogawara et al. 1991) has led some authors to conclude that the classification scheme of Pallavicini and colleagues may be too simplistic and that there is no such thing as a confined simpleloop flare, that all flares are ejective flares which exhibit essentially the same properties and can be understood with the same physical mechanism (e.g., Shibata et al. 1995; Shibata 1999).

Recently Kundu et al. (2001) suggested that several flares they analyzed could be understood as confined flares.
The basis for their conclusions was the observation of single compact sources in microwaves. However, flares can show single sources at one radio frequency but can show more complex behavior at other wavelengths indicating more complex topologies (e.g., Wang et al. 1994). It is thus difficult to make firm conclusions on whether a solar flare is confined or not based on microwave sources alone. Observations at other wavelengths such as soft X-rays, EUV or $\mathrm{H} \alpha$ are necessary to confirm the validity of such suggestions.

One approach to fully assess whether there are physically distinct categories of flares would be to carry out a thorough analysis of a large number of solar flares (preferably using observations across a wide range of frequencies). To date this has not yet been done. An alternative approach would be to examine individual flares in greater detail, as case studies, and, with an accumulated number of such analyses, be able to make a better assessment as to whether there are distinct types of flares.

With the latter viewpoint in mind, we present the results of an analysis of soft X-ray imaging observations of a solar flare which started at $\sim 15: 17$ UT on 1992 July 11. Some aspects of this flare were examined previously by Doschek et al. (1995), Khan et al. (1996), and Sylwester \& Sylwester (1999). This flare, as seen with the Yohkoh SXT was of comparatively simple geometry, consisting of two bright footpoints early in the flare and one bright loop later in the flare. In this paper we wish to examine how closely this flare compares with the supposed paradigm of a confined simple-loop flare. We focus on morphological aspects of the soft X-ray intensity, temperature and emission measure development. The data used in this work are briefly describe in Sect. 2. The observations and their analysis are presented in Sects. 3 and 4, respectively. A discussion of the results is given in Sect. 5 .

\section{Data sources}

The principal data used in this work are soft X-ray images from the Yohkoh SXT. The SXT was a grazing incidence soft $\mathrm{X}$-ray telescope, sensitive to photons in the range $(\sim 3-45 \AA)$. It provided soft $\mathrm{X}$-ray images of solar coronal plasma hotter than about $2 \mathrm{MK}$, typically taken in several different filters. Outside the times of flares, whole-Sun full-frame images (FFIs) and smaller field of view (quiet-mode) partial-frame images (PFIs) were generally taken. Usually when a large flare occurred, Yohkoh flare-mode was triggered during which high temporal and spatial resolution PFIs were taken, but no FFIs. With the SXT data it is possible to examine the morphological, temperature and emission measure development of soft $\mathrm{X}$-ray emitting plasma. In our analysis the SXT images were corrected using standard Yohkoh software and were aligned to the center of the earliest flare-mode PFI without saturation using information from the Yohkoh satellite attitude sensor (accurate to $\sim 1 \operatorname{arcsec}$ ). For the alignment of the SXT and other images we rotated the images using the expression for small magnetic features given in Howard et al. (1990).

We also examine portions of full-disk $\mathrm{H} \alpha$ line center filtergrams obtained from the heliograph of the Observatoire de Haute-Provence, France. These images provide information about the chromospheric brightenings associated with the flare. 


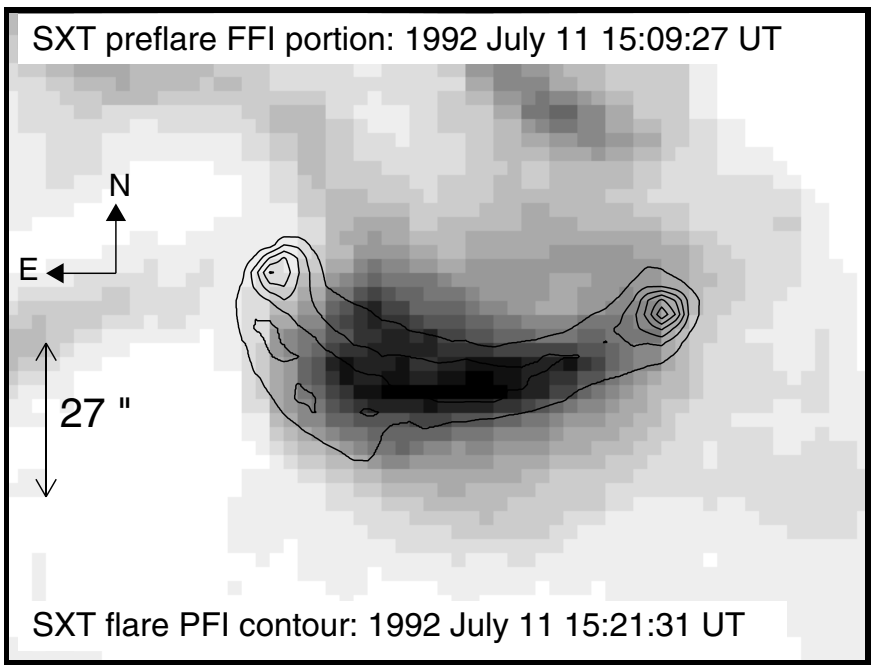

Fig. 1. The gray scale image is a portion of an SXT (Open/Al.1 filter) full-frame image (FFI) at the eventual flare region prior to the flare. Superposed on this image are equally spaced contours for an SXT flare-mode (Open/Al.1 filter) partial-frame image (PFI) early in the flare. The non-flare gray scale image was rotated to the time of the flare image.

\section{Yohkoh soft X-ray imaging data}

The duration of flare mode was comparatively long for this event and, consequently, some of the SXT taken data early in the orbit was over-written in the bubble data recorder on the Yohkoh satellite. In particular, data was lost between 15:14-15:21 UT, which includes the time of the impulsive phase of the flare. Nevertheless, the data available reveal interesting results.

\subsection{Pre-flare emission}

Examination of the Yohkoh SXT data reveals that there was only one SXT soft X-ray image which observed the flare region before the flare during the same satellite orbit in which the flare occurred. This was an FFI taken at 15:09:27 UT on 1992 July 11. The relevant portion of this image is shown as a gray scale image in Fig. 1 overlaid with an early flare-mode SXT image shown in contours. We rotated the gray scale image to the time of the flare-mode image. Although we are unable to discuss the preflare soft X-ray development in any detail, it is interesting to note that the figure indicates that there was a bright soft X-ray feature near the flare location about eight minutes before flare onset. Although the structures in the gray scale image are not well-resolved it appears that the preflare soft X-ray structures may have consisted of perhaps two bright loops "face-to-face" to each other, with their western footpoints closer to each other than their eastern footpoints.

Initial impressions of the soft X-ray data during the flare appear to show what appears to be a single soft X-ray loop (see also Fig. 4a). However, a faint soft X-ray emitting feature can be seen to the southeast in Fig. 4a. This fainter loop was noted by Doschek et al. (1995). By appropriate scaling of the images, we can demonstrate the presence of a faint loop to the south of the bright loop, as shown in Fig. 2.

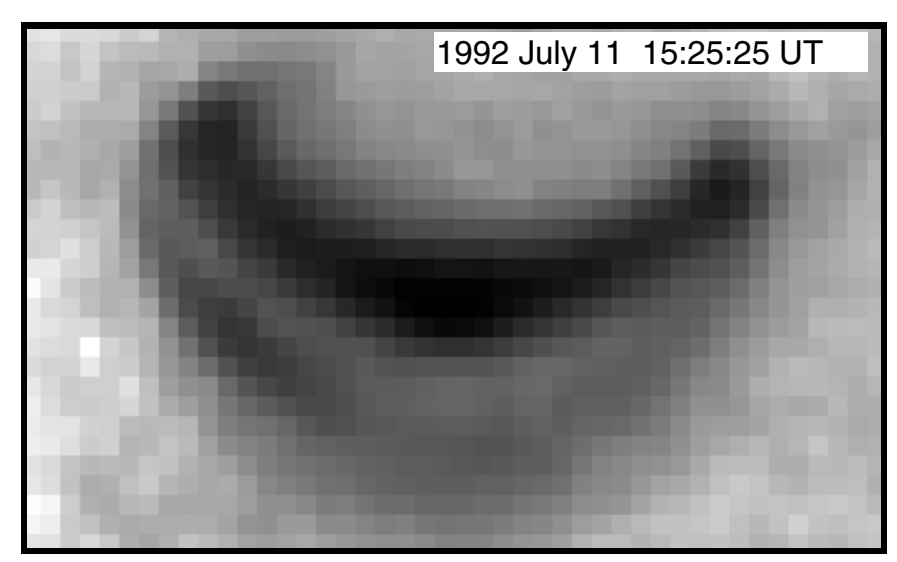

Fig. 2. A logarithmically-scaled portion of an SXT (NuDen/Al.1 filter) PFI during the flare. This indicates that the soft X-ray structure for the flare was a double-loop or "mini-arcade".

This, combined with the preflare soft X-ray image data, shows that the basic soft X-ray structure of this flare was, in fact, an arcade of at least two loops "face-to-face". Since one soft Xray loop is much brighter than the other during the flare, this indicates that the bulk of the flare energy deposition occurred in that loop. However, the overall flare structure was clearly not that of a simple single loop configuration.

The presence of bright footpoints early in the flare presents another striking impression. This is consistent with the idea of the ablation of chromospheric material at the loop footpoints into loops increasing the coronal density during the impulsive phase of the flare.

\subsection{Flare evolution}

During flare-mode images were taken of the flare with several different soft X-ray filters as well as with different effective fields of view and spatial resolutions. The widest field of view images have the lowest spatial resolution. Such images are often useful for showing faint features such as ejecta moving away from the flare location. However for this particular event no clear ejecta were observed. The smallest field of view images, which have the highest spatial resolution, are the most useful for this event. During the flare, the soft X-ray structures were sufficiently large in spatial extent to allow detailed examination of the development of the spatially resolved features. We now present the results of an examination of some aspects of the evolution of the soft X-ray structures. We concentrate on the brighter of the two loop structures discussed in Sect. 3.1.

\subsubsection{The flare-loop cross-section}

A movie of a sequence of soft X-ray images (normalized by exposure duration) during the flare suggests that the flare loop apex gets broader with time. Figure 3 shows two soft X-ray images, one early in the flare and one later in the flare. The images have been scaled to show the relative differences between the images. These images indicate an apparent broadening of the top of the main flare loop with time. In order to investigate the variation of the flare loop cross-section further we 


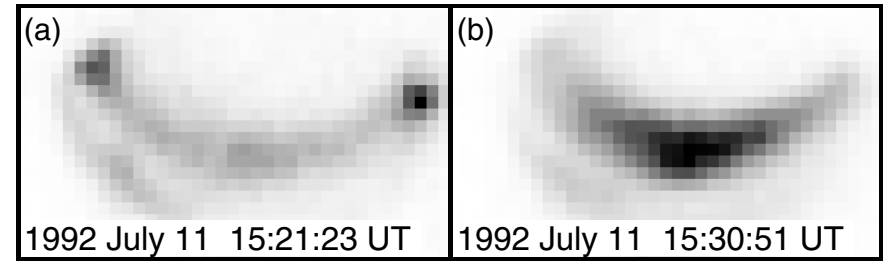

Fig. 3. Two exposure normalized SXT (NuDen/Al.1 filter) images, one taken early in the flare a), and the other taken later in the flare b), indicating an apparent broadening of the loop at its top with time. Note the bright footpoints in panel a).

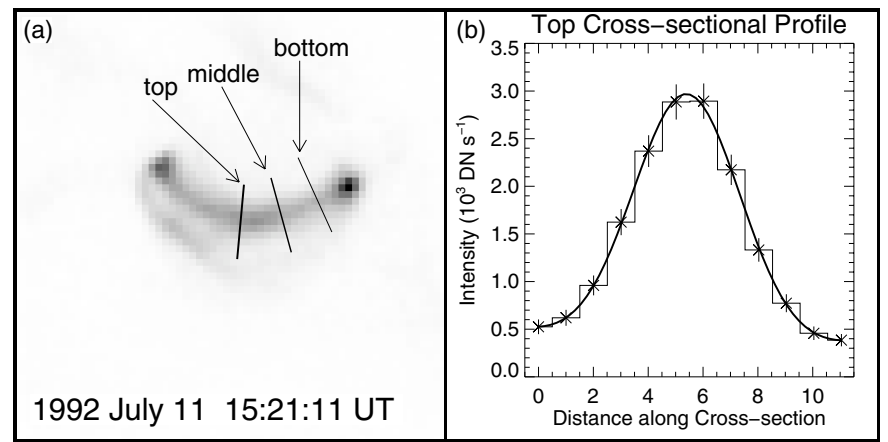

Fig. 4. a) A portion of an SXT (Open/Al12 filter) image early in the flare indicating the locations of "top", "middle", and "bottom" crosssectional "cuts" across the western leg of the flare loop. b) The values (cross marks and histogram) and uncertainties along the crosssectional cut at the top of the loop for the image shown in a), along with a fitted line to the data.

examined the intensity profile along cross-sectional "cuts" at locations we loosely term the "top", "middle", and "bottom" of the (west) leg of the flare loop. Figure 4a shows the crosssectional cuts used on an SXT image, while Fig. 4b shows an example of the values of the intensity at points along the crosssectional cut at the top of the loop for that image. We find that a function of the form of a Gaussian term plus a quadratic expression (to fit the background) provides a reasonable fit to the intensity, $I$, along the cuts:

$I(x)=a_{0} \mathrm{e}^{-z^{2} / 2}+a_{3}+a_{4} x+a_{5} x^{2}$,

where $x$ is the distance along the cross-sectional cut (starting from the most southerly end), $z=\left(x-a_{1}\right) / a_{2}$, and $a_{0}, a_{1}, a_{2}$ are the height, center and width of the Gaussian term, respectively, while $a_{3}, a_{4}, a_{5}$ are the coefficients for the constant, linear and quadratic terms respectively. Also shown in Fig. 4b is the best fit (thick line) to the data assuming a function of the form of Eq. (1).

In Fig. 5 we plot the best fits to the middle crosssectional cut for each Open/Al12 filter flare-mode PFI from 15:21:27-15:27:55 UT. A point of interest highlighted by this figure is the appropriate way the cross-sectional width of the loop should be defined. If we choose a threshold value (e.g., $7 \times 10^{4} \mathrm{SXT}$ data numbers (DN) s${ }^{-1}$ ) to define the width of the loop, then we would conclude that the loop did broaden significantly during the flare. However, if we consider the Gaussian width as characterizing the cross-sectional width then we would conclude that there was, in fact, little significant
Gaussian Fits to Middle Cross-sectional Profiles

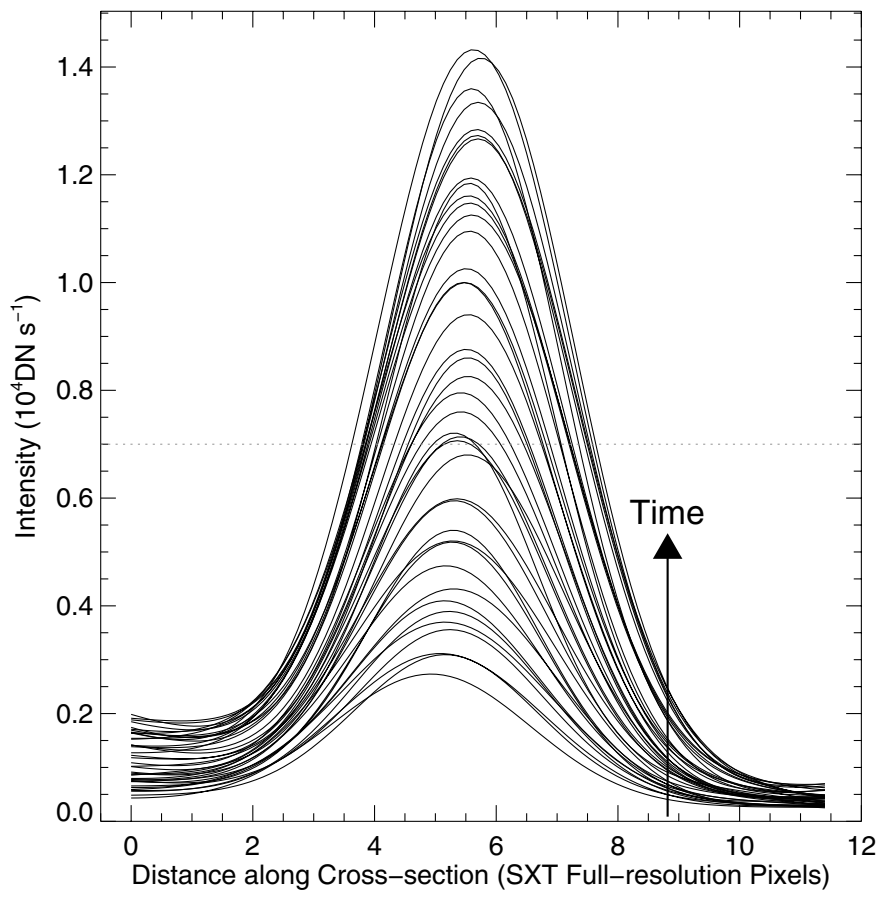

Fig. 5. The fitted curves to the values of the soft X-ray intensity along the middle cross-sectional cut for SXT Open/Al12 filter images in the interval 15:21:27-15:27:55 UT on 1992 July 11.

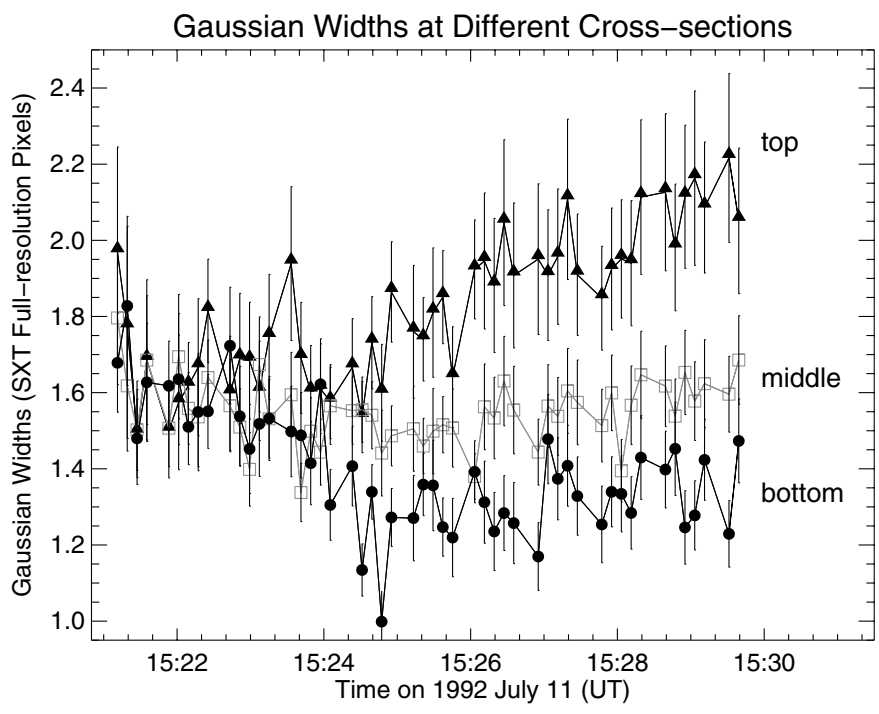

Fig. 6. The widths of the Gaussian terms in the fits to the values of the soft X-ray intensity along the different cross-sectional cuts of the loop as a function of time.

change in the loop cross-section for the cross-sectional cut and interval considered in Fig. 5. We believe that the Gaussian width (i.e., the full width at half maximum of the Gaussian term) is the physically more meaningful description of the cross-sectional width. Thus, despite the appearance of the sequence of images, we arrive at the conclusion that there was little significant change in the middle cross-sectional width of the flaring loop with time.

In Fig. 6 we plot the variation of the fitted Gaussian width with time for the top, middle and bottom cross-sectional cuts. 


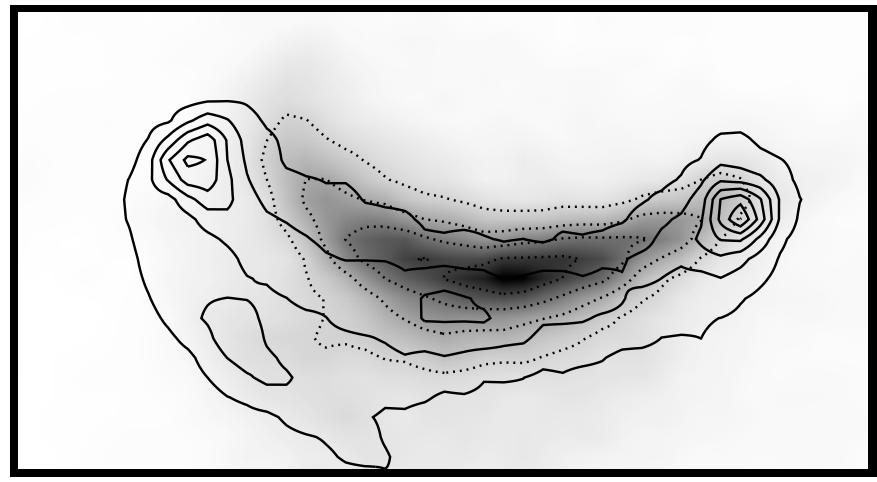

Fig. 7. The gray scale image is a portion of a NuDen/Al.1 SXT PFI taken late in the flare (at 15:44:49 UT). Superposed on this image are equally spaced contours (dotted lines) of that same image, as well as contours (thick solid lines) for a NuDen/Al.1 SXT image taken early in the flare (at 15:21:23 UT). The displacement of the contours between these times indicates an apparent motion of the flare loop.

(We believe the pseudo-oscillatory fluctuations in the values of the coefficients describing the Gaussian term are due to the pixelization of the images.) We find little significant change in the cross-sectional width of the loop at the bottom and middle cuts when one considers the Gaussian width as characterizing the loop width. But Fig. 6 indicates that eventually the Gaussian width of the top of the loop increases with time. Despite the stricter requirements we have imposed by using the Gaussian width (as opposed to a threshold value), we do find an increase in the cross-sectional width of the loop at the top cross-sectional cut of $\sim 30 \%$ from its initial value. Klimchuk et al. (1992) examined active region (but not flaring) coronal loops seen in Yohkoh SXT images and found that they appeared to have nearly constant cross-sectional radii. They found that the cross-sectional variation of coronal loops varied by only about 10-20\% along their lengths. Figure 6 indicates a significantly larger variation $(\sim 50 \%)$ for times near 15:29 UT for this flare. Klimchuk (2000) carried out a more detailed study using higher resolution data and concluded that coronal loops seen with the Yohkoh SXT show typical increases in cross-sectional width from footpoint region to loop-top region of $\sim 30 \%$.

\subsubsection{Apparent motion of the flare loop}

In Fig. 7 we show a gray scale SXT image late in the flare overlaid with its own contours (dotted lines) and contours (solid lines) of an SXT image taken early in the flare. The figure indicates an apparent "inward" motion of the loop with time. That is, apparent motion toward the center of curvature of the loop, as seen in projection. This apparent motion was first noted by Doschek et al. (1995).

To demonstrate this apparent motion we plot the locations of the centers of the Gaussian terms in the fits (to the soft $\mathrm{X}$-ray intensity values of the cross-sectional cuts) with time in Fig. 8. The relative change in location of the centers for a given cross-sectional cut with time is the main feature we draw attention to in this plot. The figure indicates that the top, middle and bottom parts of the loop all move inward (i.e., toward the projected center of curvature of the loop) with time.

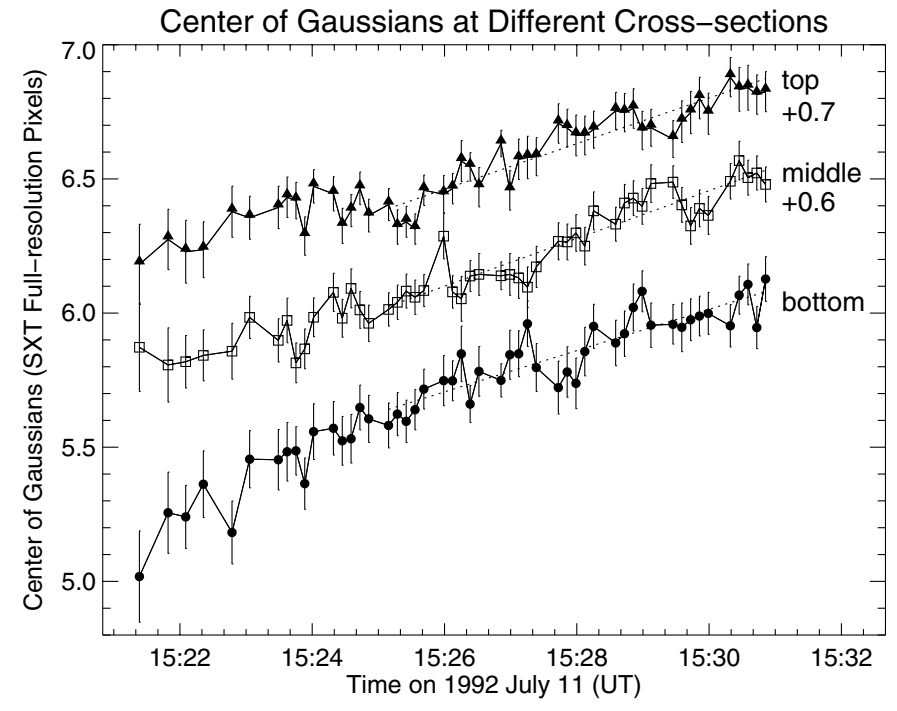

Fig. 8. The positions of the centers of the Gaussian terms in the fits to the cross-sectional cuts of the loop (in NuDen/Al.1 SXT images) as a function of time. The positions of the points for the top and middle cross-sectional cuts are offset by 0.7 and 0.6 pixels, respectively, for clarity.

Performing a least-squares linear fit to values over the interval of 15:25:09-15:30:51 UT, we find that the apparent speeds of motion along the bottom, middle, and top cuts of the loop are $2.3 \pm 0.2,2.7 \pm 0.2$, and $2.6 \pm 0.2 \mathrm{~km} \mathrm{~s}^{-1}$. Note that typical observed speeds of flows in the photosphere are of order $1 \mathrm{~km} \mathrm{~s}^{-1}$. Since the magnitude of the speeds we observe is greater, the apparent motion we measure is not likely to have originated from photospheric motions at the footpoints of a coronal loop. In general, we would not expect photospheric motions to result in motions in nearly the same direction for both footpoints.

Movies of the SXT soft X-ray images indicate that the motion of the main flare loop is more pronounced on the eastern leg compared to the western leg. This finding is apparent from the greater displacement of the eastern leg of the loop observed later in the flare, as seen in Fig. 7. The results presented above are for the western leg of the loop. We would expect higher speeds of motion for the eastern leg.

\subsubsection{Peak intensity at the loop cross-sectional cuts}

In Fig. 9 we plot the height of the Gaussian terms in the fits for different cross-sectional cuts of the main flare loop. This indicates that the peak values of the intensity for the top, middle and bottom cross-sectional cuts all increase with time up to the peak of the soft X-ray flare. Performing a least-squares linear fit to values over the interval of 15:21:05-15:30:35 UT on 1992 July 11, we find that the peaks of the Gaussian fits along the bottom, middle, and top cuts of the loop increase at the rates of $187 \pm 5,341 \pm 6$, and $478 \pm 15 \mathrm{DN} \mathrm{s}^{-1}$, respectively. Thus, the rates of increase of the peaks of the soft X-ray intensity values along the legs of the flare loop are roughly proportional to the distance from the bottom of the loop (or, alternatively, inversely proportional to the distance from the top of the loop). 


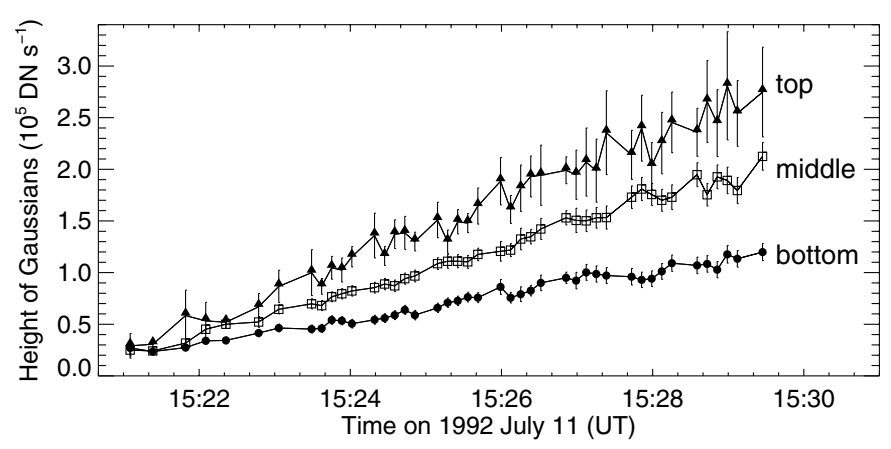

Fig. 9. The peak values of the Gaussian terms in the fits to the crosssectional cuts of the loop (in NuDen/A1.1 SXT images) as a function of time. The figure shows that the soft X-ray intensity along the loop is roughly proportional to the distance along the loop from the footpoints.

\subsubsection{Temperature and emission measure development}

Using the ratio of the values of the pixels in SXT images taken with different filters we constructed temperature and emission measure maps of the flare. The images were taken with three different soft X-ray filters: Al $1265 \AA$ (Al.1), Al $11.6 \mu \mathrm{m}$ (Al12), and Be $119 \mu \mathrm{m}$ (Be119). The filter ratio Al12/Al.1 is generally double valued and not useful for determining the temperature and emission measure in this event. The filter ratios Be119/Al.1 and Be119/Al12 are useful. To illustrate our results we present results from an analysis using the Be119 and Al12 images. Although not detailed here we find qualitatively similar results using the Be119 and Al.1 images.

We first interpolated the images taken with the Al12 filter to the times of those taken with the Be119 filter. In order to improve the statistics, and hence the quality of the maps, we created accumulated images for each filter by adding eight consecutive images before computing the values for the filter ratio on a pixel by pixel basis. For each accumulated image pair we obtained a single temperature map and a single emission measure map. The accumulated images, and hence the temperature and emission measure maps, cover the interval 15:20:57-15:54:25 UT on 1992 July 11. In Fig. 10a we show an emission measure map early in the flare (along with the time interval for the images used to make the map), and the location of a cross-sectional cut. The cut shown here is similar to the top cross-sectional cut for the soft X-ray intensity shown in Fig. 4a, except that it extends further north. Figure 10b shows the variation of the emission measure values at points along the cross-sectional cut. We are unable to easily find fitted functions of the form used to describe the soft X-ray intensity for this and for subsequent cross-sectional profiles, so we simply plot the derived values without attempting any fit.

In Fig. 11 we plot the emission measure values at each pixel along the top cross-sectional cut for each emission measure map. The lines and set of points for the cuts for each map are drawn such that chronologically successive profiles are shown in progressively thicker lines. The uncertainties in the emission measure values are much smaller than the $\times$ symbols drawn. A number of aspects of the emission measure evolution are

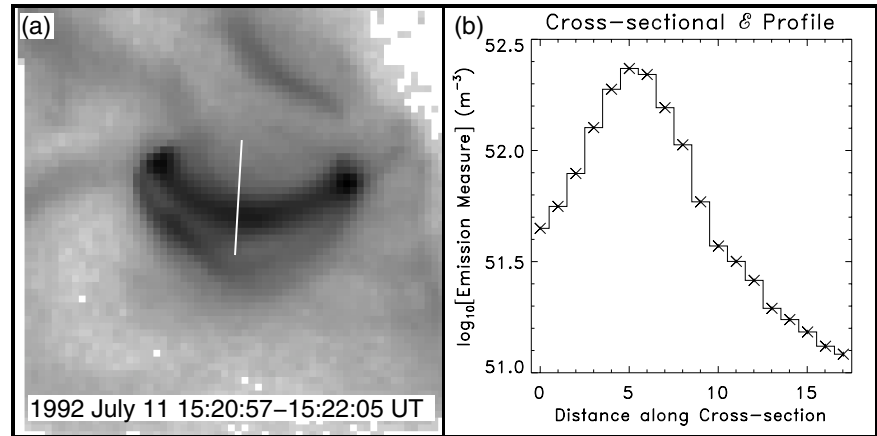

Fig. 10. a) A portion of a (logarithmically-scaled) SXT emission measure map early in the flare indicating the location of the top crosssectional cut of the loop used in some of the emission measure and temperature analysis. b) The emission measure values for each SXT full-resolution pixel along the top cross-sectional cut for the map shown in a).

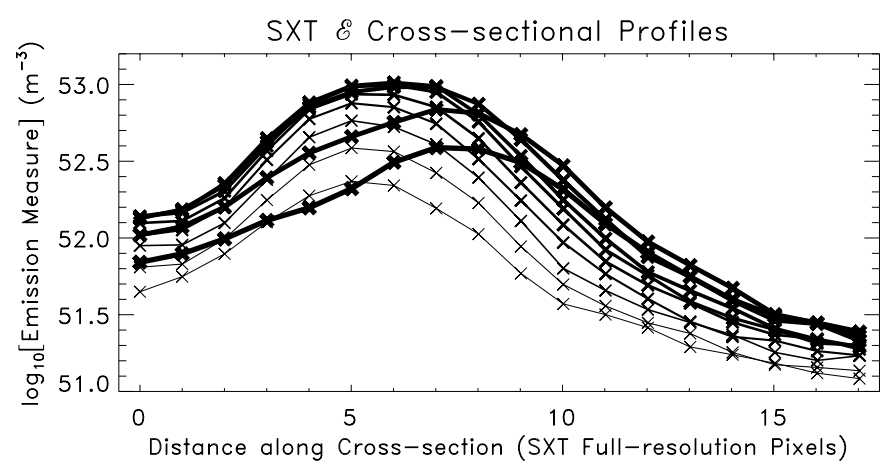

Fig. 11. The values of the emission measure $(\mathcal{E})$ at each pixel along the top cross-sectional cut of the loop at different times. Each set of data and fits, representing a single constructed emission measure map, are drawn such that later times have progressively thicker symbols and lines than the previous set.

apparent from the figure. The peak value of the emission measure of the loop initially increases with time, and is located at approximately the same location during the increase. Then, as the emission measure decreases the location of the peak in the emission measure moves inward with time. Furthermore, the plots show that the cross-section of the soft X-ray emitting region gets broader with time.

For the temperature maps we find that the values along the top cross-sectional cut for each image in the sequence are also not easy to fit with a simple function. Consequently, we simply plot the temperature values along the cut for each map in Fig. 12 and draw chronologically successive profiles in progressively thicker lines. The error bars are about the size of the circle symbols or smaller. The peak value of the temperature is observed to decrease with time. The location of the peak temperature region also moves inward with time. In addition we find that the approximate location of the peak in the temperature early in the flare, later becomes the location of a trough in the temperature.

In Fig. 13 we show gray scale images of the temperature taken at four different time intervals, two during the rise of the soft X-ray flux and two during the decay of the soft Xray flux. The contours shown in dotted black lines in Fig. 13 


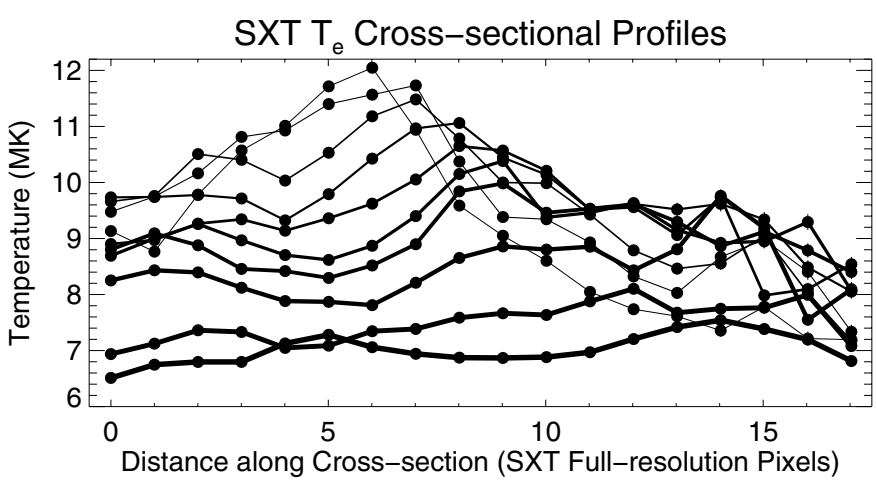

Fig. 12. The values of the temperature at each pixel along the top cross-sectional cut of the loop at different times. Each set of data and fits, representing a single constructed temperature map, are drawn such that later times have progressively thicker symbols and lines than the previous set.

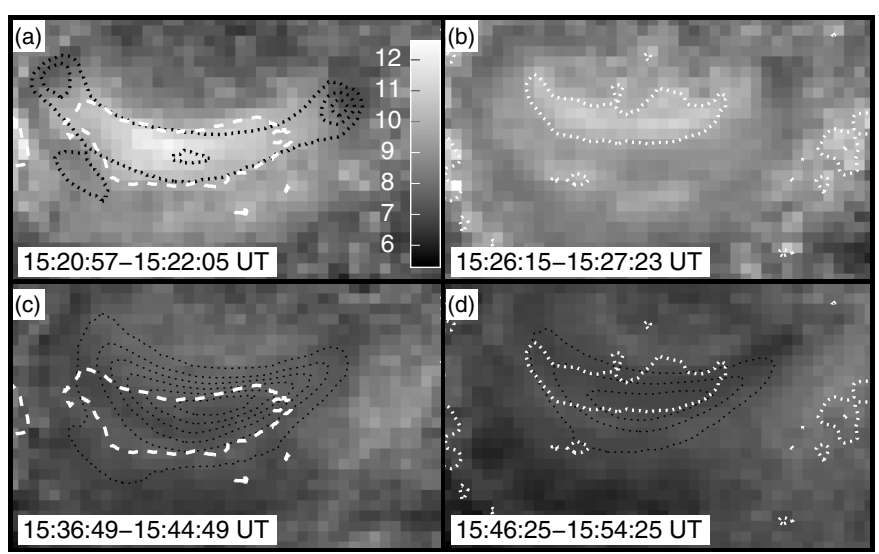

Fig. 13. A sequence of SXT temperature maps showing hot loop regions which later become cool loop regions. The maps were made by taking the ratio of different filter SXT images summed over the time intervals shown at the bottom of each panel. The gray scale bar in a) indicates the values of the temperature in $\mathrm{MK}$ for all of the panels (i.e., apparent differences between the maps are real).

are for the corresponding emission measure maps. In Fig. 13a we also show a color bar which shows the temperature values in MK for all the gray scale images shown in the figure. On the images taken early in the flare (i.e., Figs. 13a and b) we have over-plotted (in white broken lines) contour levels illustrating the high temperature regions. The temperature maps later in the flare (i.e., Figs. 13c and d) show that these locations then become locations of a dip in the temperature relative to the surrounding areas. To our knowledge such an effect has not been reported previously. The contrast of the flaring loop temperatures with the temperatures of the surrounding quiescent corona shows that the flare loop did not decay into a typical bright active region loop, but to a cooler one. Note that Figs. 13a and $b$ also show the apparent northward motion of the high temperature region with time.

\section{4. $\mathrm{H} \alpha$ observations}

The flare, of optical importance $\mathrm{SN}$, was observed in $\mathrm{H} \alpha$ by the heliograph of the Observatoire de Haute-Provence, France with

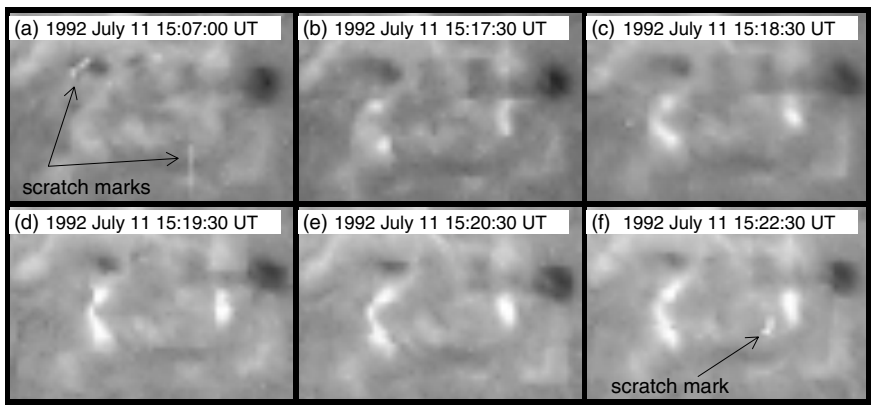

Fig. 14. Portions of full-disk $\mathrm{H} \alpha$ filtergrams from the Observatoire de Haute-Provence before and during the flare examined in this paper. Each image is individually (linearly) scaled to span the full range of display values to emphasize the most important features in each image. The images show the $\mathrm{H} \alpha$ brightenings associated with the flare.

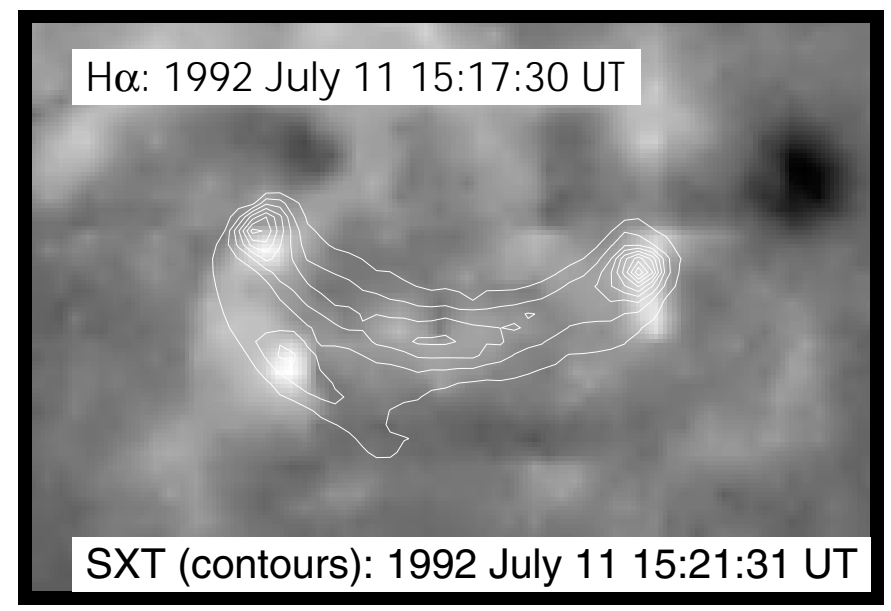

Fig. 15. The gray scale image is the $\mathrm{H} \alpha$ image from the Observatoire de Haute-Provence shown in Fig. 14c. Superposed on this image are equally spaced contours for an SXT soft X-ray image during the flare.

a cadence of about one minute. The $\mathrm{H} \alpha$ images show brightenings in the form of brilliant flaring points, approximately centered at heliocentric coordinates S13E03. A sequence of portions of $\mathrm{H} \alpha$ filtergrams from the heliograph of the Observatoire de Haute-Provence are shown in Fig. 14.

Prior to the flaring, the sunspot region did not possess a prominent active region filament; several, small, faint filaments were visible, but none in the immediate vicinity of the later flaring region. An example of the preflare region is shown in Fig. 14a.

Based on the $\mathrm{H} \alpha$ images the flaring started at about $\sim 15: 17.5$ UT with the appearance of the brilliant flaring points. The brilliant $\mathrm{H} \alpha$ flaring features are likely to be due to emission at the footpoints of coronal loops. In Fig. 15 we show the $\mathrm{H} \alpha$ image shown in Fig. 14b with overlaid SXT image contours, which strongly supports this view. This figure also indicates that the early bright $\mathrm{H} \alpha$ feature to the south of the eastern footpoint was likely to have been the footpoint of the adjacent faint "parallel" soft X-ray loop.

The $\mathrm{H} \alpha$ data indicates that the emission to the east consisted of perhaps two or three bright points, while the emission 
to the west shows only one bright point. Note also that some of the SXT images indicate that the western footpoints of the soft X-ray loops are closer together compared to the eastern footpoints. As the flare progressed the $\mathrm{H} \alpha$ patches of emission spread in a roughly north-south direction. The bright $\mathrm{H} \alpha$ features associated with the eastern footpoints appear to become connected and appear almost ribbon-like, suggesting a series of coronal loops rather than one or two coronal loops. The $\mathrm{H} \alpha$ features also indicate a greater displacement of the eastern footpoints compared to the western footpoints of the presumed loops.

The $\mathrm{H} \alpha$ images are taken in line center with the narrow band-width of $\sim 0.75 \AA$. Consequently, it is impossible to detect motions with accelerations higher than $0.3 \mathrm{~km} \mathrm{~s}^{-2}$ and no surge associated with the flare was seen in that set of data. No filament disappearance was observed to be associated with this flare either.

\section{Conclusions}

We examined SXT images for a solar flare which occurred at $\sim 15: 17$ UT on 1992 July 11. The SXT images show two bright footpoints early in the flare and a single bright loop later in the flare. While the bright loop is the dominant source of emission we find that closer examination of the soft X-ray structures (both prior to the flare and during the flare) reveals that the flare actually consisted of at least two loops almost face-to-face. Using the highest spatial resolution SXT images we then examined the brightest flaring loop to understand the flare development.

We examined the SXT intensity profiles across the loop at various locations along the loop. We considered the width of the loop to be characterized by the width of a Gaussian fit to the profile. This stricter-than-usual definition of the loop width leads us to conclude that the cross-sectional width at the bottom of the loop and halfway up the loop leg do not change significantly with time. This is contrary to the impression derived from the appearance of a movie of a sequence of SXT images (which implicitly infers the loop width to be determined by intensity values above a fixed threshold value). Nonetheless, despite this stricter definition of loop width we do find significant broadening at the top of the loop with time. The looptop region shows a broadening of $\sim 30 \%$ compared to its initial value. We also find that the variation of the cross-sectional width from bottom to top of the loop to be as much as $\sim 50 \%$. This is larger than the variation reported by Klimchuk et al. (1992) and Klimchuk (2000).

Examination of SXT images indicate a slow apparent motion approximately transverse to the loop major axis in a northward direction. This is confirmed quantitatively from examination of the cross-sectional profiles along the loop. We find speeds of motion of about $2.5 \mathrm{~km} \mathrm{~s}^{-1}$ at various points along the loop. These speeds are greater than typical speeds of photospheric flows and consequently we conclude that the observed motion was not likely to have been due to real motions in the corona related to photospheric motions. Instead we infer the motions to be merely apparent motions caused by the

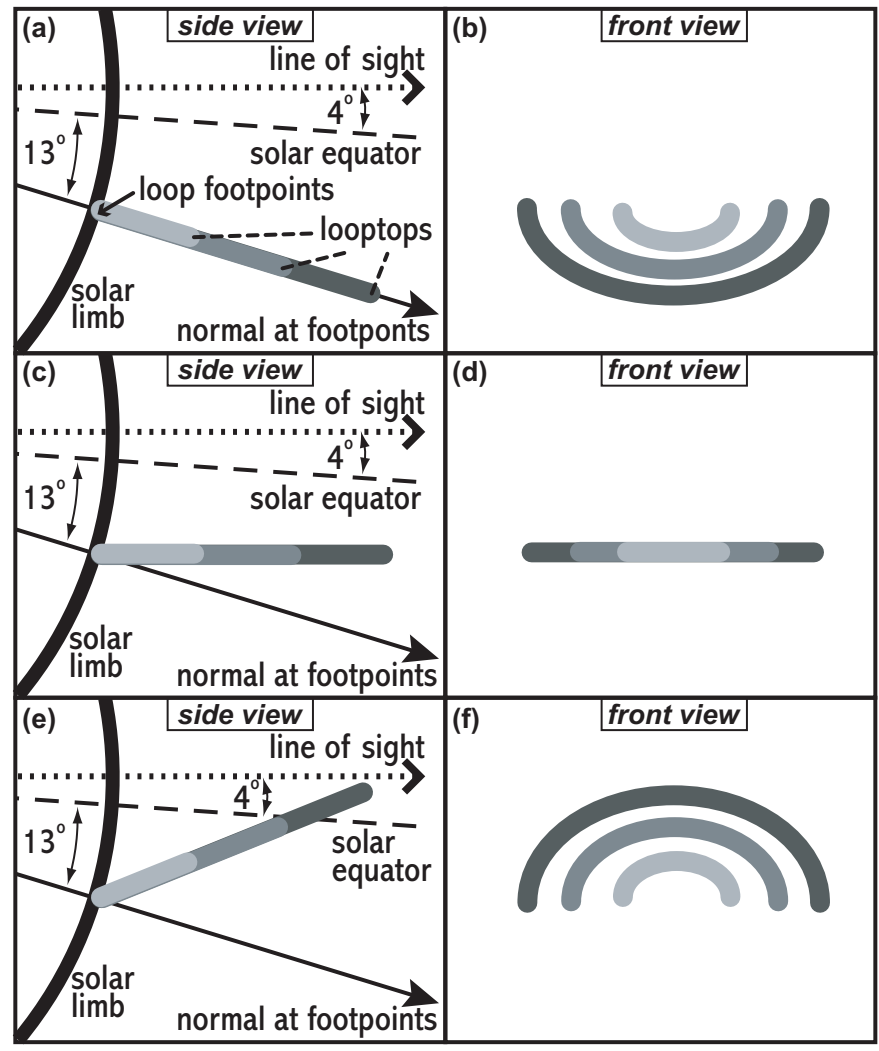

Fig. 16. A schematic diagram illustrating the how the brightening of magnetic loops (later loops are shown darker) all located in a plane (with different possible orientations) cannot explain the observations of the motion of the flare loop and the broadening of its loop-top region.

brightening in soft X-rays of adjacent magnetic field lines which brighten in succession.

However the apparent motion requires further consideration and explanation. In the usual picture of magnetic reconnection associated with a solar flare (e.g., Priest \& Forbes 2000) magnetic field lines which are located further out, with progressively wider separated footpoints and higher apices, are successively reconnected. Thus normally such a scenario would result in soft X-ray features which appear to expand in height and in footpoint separation.

If we suppose a similar scenario occurs in the flare event considered here then how do we understand the apparent northward motion? Consider the location of the flare on the Sun as well as its initial loop configuration. The flare was located at S13 in heliocentric coordinates. The SXT software corrects for the position angle of the solar rotation axis, but not the inclination of the solar equator to the line of sight. At the time of this event this inclination was $4^{\circ}$ to the south. Thus the effective inclination of the latitude of the flare was $-17^{\circ}$. The simplest picture of magnetic structures stacked higher in altitude and all located in a plane encounters problems explaining the observations. Various geometrical scenarios with successive loops located within a plane are illustrated in Fig. 16. In such a scenario with the constraint of magnetic structures all located in a plane requires the magnetic field lines to be tilted 


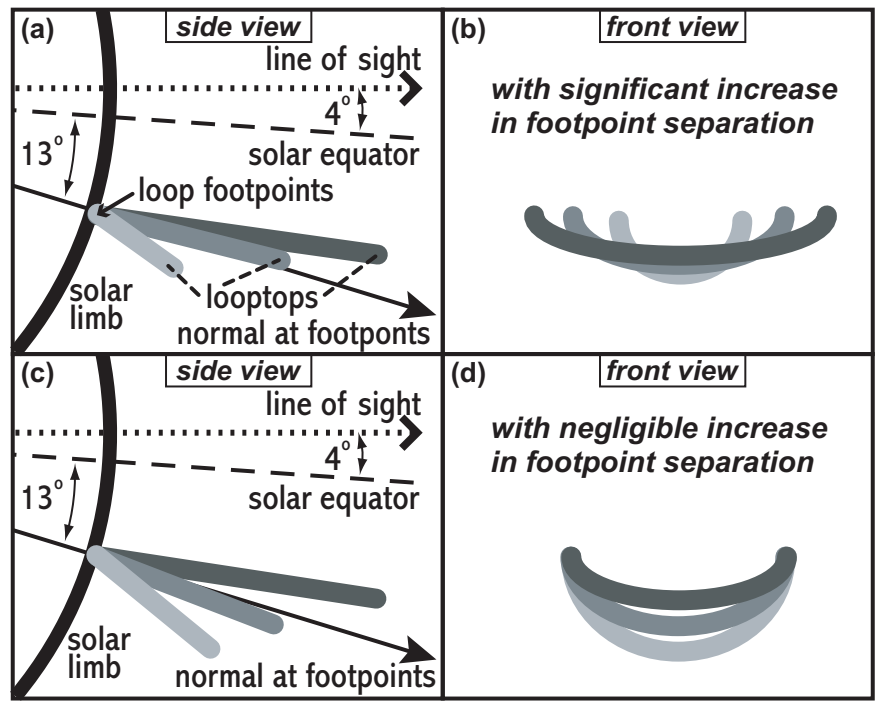

Fig. 17. A schematic diagram illustrating the how the brightening of loops lying tilted to the south of the line of sight but in successively less tilted planes and with relatively close footpoint separations can explain the observations of the apparent motion of the flare loop and the broadening of its loop-top region.

by more than $17^{\circ}$ north of the normal at the flare location in order to observe northward motion. However the soft X-ray loop observed throughout the flare duration appears to have its projected center of curvature to the north. This observation requires that the soft X-ray loop structures must have remained tilted to the south of the line of sight of Yohkoh.

It is therefore necessary for successively reconnected magnetic field lines to lie in successively less tilted planes (but all still tilted to the south of the line of sight). This however is still not sufficient. In order to give rise to the apparent motion the footpoint separation of successive field lines must remain relatively close together. This is illustrated in Fig. 17.

There is one further constraint required to ensure that successive apices appear to lie further north with time. This is illustrated in Fig. 18. The apparent velocity of the loop-top region must be directed to the north of the line of sight. Also, if we suppose that successive loops are at greater heights then these conditions require that the velocity of loop arcade growth must lie in the range of directions between the vectors $\boldsymbol{v}_{\mathrm{t}}$ and $\boldsymbol{n}_{1}$, which represent a velocity wholly along the tangent to the line joining the loop-top to the footpoint and a vector along the line of sight, respectively. Thus $v_{\mathrm{t}}$ is the minimum possible velocity. Arcade growth velocity vectors at the other possible directions must be greater than $v_{\mathrm{t}}$ in order to give the observed loop motion transverse to the line of sight, $v_{0}$. The schematic diagram indicates that $v_{\mathrm{t}}=v_{\mathrm{o}} \sec \phi$, where $\phi$ is the inclination of the loop to the line of sight. For simplicity, assuming that the initial loop lies along the normal to the solar surface then $\phi=17^{\circ}$ and using $v_{\mathrm{o}} \sim 2.6 \mathrm{~km} \mathrm{~s}^{-1}$ implies $v_{\mathrm{t}} \sim 2.7 \mathrm{~km} \mathrm{~s}^{-1}$. This value represents a lower limit on the actual speed. Such speeds are consistent with those typically observed in arcade flare development (e.g., Dizer 1978).

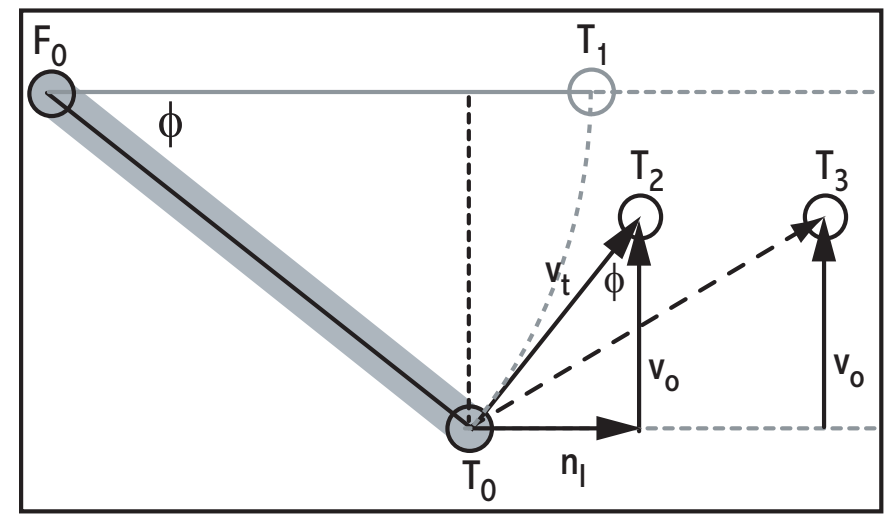

Fig. 18. A schematic diagram illustrating the constraint on the velocity vector of apparent loop-top motion. $F_{0}$ represents the loop footpoint while $T_{0}-T_{3}$ represent possible loop-top locations. $n_{1}$ represents a vector wholly along the line of sight, while $v_{\mathrm{t}}$ represents a velocity vector wholly along the tangent to the line joining the loop-top region to a footpoint. The velocity vector of apparent loop-top motion must lie somewhere between the directions of these vectors.

Note also that the loops of some arcade flare events seen in $\mathrm{H} \alpha$ can show large inclinations to the local vertical (e.g., Bruzek 1964). Coronal data also show that some active regions consist of loops which are increasingly inclined towards the outer parts of the active regions (e.g., Gary \& Alexander 1999; Aschwanden et al. 2000). Thus the relevant magnetic field structures for this particular flare probably consisted of what might be termed a splayed arcade structure

A variant of the scenario mentioned above would be that the reconnection proceeds laterally. That is, the magnetic field lines which brighten in succession did not have higher apices compared to previously reconnected field lines, but instead were adjacent to each other with their loop tops lying on a circular arc of fixed radius (i.e., the dashed gray arc shown in Fig. 18).

The observations establish the broadening of the looptop region as the flare progresses, as based on our objective characterization of loop widths with Gaussian fits. This apparent broadening may be due to actual broadening indicating a change to a more potential magnetic configuration resulting from the release of magnetic energy during the flare. (This follows since the apex of a potential loop is broader than its footpoints, e.g., Klimchuk 2000.) The alternative scenario described above and illustrated qualitatively in Figs. $17 \mathrm{c}-\mathrm{d}$ appears to suggest that the broadening could be merely an apparent broadening due to the brightening of adjacent loops.

We conclude that the scenario outlined above of brightening loops in what might be termed a splayed arcade structure can naturally explain the apparent northward motion of the flare loop as well as the apparent broadening of the loop-top region. However it does not explain why one leg of the loop appears to move more than the other. Perhaps this is due to differences in the magnetic field concentrations at the footpoints.

We also examined the temperature and emission measure development of the soft X-ray flaring loop. This was done by examining constructed SXT emission measure and temperature images as well as the loop-top cross-sectional profiles of such 
images. The cross-sectional profiles of the temperature and emission measure near the loop-top region of the flare show an apparent motion of the high temperature and emission measure regions with time, similar to that observed with the soft X-ray intensities. This motion is also apparent from the constructed SXT emission measure and temperature images themselves.

From the cross-sectional profiles of the loop-top temperature shown in Fig. 12 and from temperature maps shown in Fig. 13 this flare has allowed us to notice a "cool loop" phenomenon where the hot flaring loops later become over-cooled loops. That is, the flare loops remain bright in the late phase but have temperature deficits relative to the neighboring active region structures. Given the possible identification of the flare loops with previously existing hot active region loops (see Fig. 1), how can this interesting observation be explained? It appears to suggest either the equilibrium state within the flaring structures was modified to the state of a loop cooler than an active region loop or the flare cooling mechanism was more efficient than whatever heating mechanism existed inside the loop (be it flare or regular active region heating) leading to a lower temperature state. The reason for the transformed equilibrium state of the loops or the increased efficiency of the cooling is unclear but may have been associated with the huge increase in particle number density caused by the ablation of chromospheric plasma into the coronal loop during the flare impulsive phase or the altered magnetic state (expected from the flare energy release).

While the initial $\mathrm{H} \alpha$ development consisted of brilliant points, as the flare develops the emission became more ribbonlike, strongly suggesting a small arcade structure.

The flare did not show a clear eruptive signature in SXT images. However, since there are no SXT observations during the crucial time interval of the impulsive phase of the flare it is not possible to be definitive about the lack of an eruptive signature in soft X-rays. Nonetheless, no filament eruptions or other eruptive signatures were seen in the $\mathrm{H} \alpha$ line center emission from the heliograph of the Observatoire de Haute-Provence nor reported by other observatories in Solar Geophysical Data. Despite the flare's favorable location near the central meridian it does not appear to have been a geo-effective event. Altogether these suggest that the flare was not a strongly eruptive event.

We conclude that while the flare exhibited the characteristic of the lack of clear eruptive signatures, it exhibited several other characteristics suggesting that this flare does not fit the paradigm of a confined simple-loop flare. The results of our analysis indicate that this flare may instead be better understood as a mini-arcade flare.

Acknowledgements. J.I.K. acknowledges financial support from the Academy of Finland. H.S.H. was supported under NASA contract NAS 8-40801.

\section{References}

Acton, L. W., Finch, M. L., Gilbreth, C. W., et al. 1980, Sol. Phys., 65, 53

Aschwanden, M. J., Alexander, D., Hurlburt, N., et al. 2000, ApJ, 531, 1129

Bohlin, J. D., Frost, K. J., Burr, P. T., Guha, A. K., \& Withbroe, G. L. 1980, Sol. Phys., 65, 5

Bruzek, A. 1964, ApJ, 140, 746

Culhane, J. L., Acton, L. W., \& Gabriel, A. H. 1984, Mem. Soc. Astron. Ital., 55, 673

Dizer, M. 1978, Sol. Phys., 59, 357

Doschek, G. A., Strong, K. T., \& Tsuneta, S. 1995, ApJ, 440, 370

Gary, G. .A., \& Alexander, D., 1999, Sol. Phys., 186, 123

Gold, T., \& Hoyle, F. 1960, MNRAS, 120, 89

Heyvaerts, J., Priest, E. R., \& Rust, D. M. 1977, ApJ, 216, 123

Howard, R. F., Harvey, J. W., \& Forgach, S. 1990, Sol. Phys., 130, 295

Kahler, S. W., Sheeley, N. R. Jr., \& Liggett, M. 1989, ApJ, 344, 1026

Kay, H. R. M., Harra, L. K., Matthews, S. A. Culhane, J. L. \& Green, L. M. 2003, A\&A, 400, 779

Khan, J. I., Hudson, H. S., Sterling, A. C., \& Lemen, J. R. 1996, in Magnetic Reconnection in the Solar Atmosphere, ed. R. D. Bentley, \& J. T. Mariska (San Francisco: Astronomical Society of the Pacific), ASP Conf. Ser., 111, 162

Klimchuk, J. A. 2000, Sol. Phys., 193, 53

Klimchuk, J. A., Lemen, J. R., Feldman, U., Tsuneta, S., \& Uchida, Y. 1992, PASJ, 44, L181

Kundu, M. R., White, S. M., Shibasaki, K., Sakurai, T., \& Grechnev, V. V. 2001, ApJ, 547, 1090

Masuda, S., Kosugi, T., \& Hudson, H. S. 2001, Sol. Phys., 204, 55

Melrose, D. B. 1997, ApJ, 486, 521

Nishio, M., Yaji, K., Kosugi, T., Nakajima, H., \& Sakurai, T. 1997, ApJ, 489, 976

Ogawara, Y., Takano, T., Kato, T., et al. 1991, Sol. Phys., 136, 1

Pallavicini, R., Serio, S., \& Vaiana, G. S. 1977, ApJ, 216, 108

Pallavicini, R. 1982, Mem. Soc. Astron. It., 53, 461

Pallavicini, R. 1991, Phil. Trans. R. Soc. Lond. A, 336, 389

Pearce, G., \& Harrison, R. A. 1988, A\&A, 206, 121

Priest, E. R., \& Forbes, T. G. 2000, Magnetic Reconnection: MHD Theory and Applications, (Cambridge, UK: Cambridge Univ. Press)

Sakai, J.-I., \& de Jager, C. 1996, Space Sci. Rev., 77, 1

Shibata, K. 1999, Ap\&SS, 264, 129

Shibata, K., Masuda, S., Shimojo, M., et al. 1995, ApJ, 451, L83

Spicer, D. S. 1977, Sol. Phys., 53, 305

Sylwester, B., \& Sylwester, J. 1999, Acta Astron., 49, 85

Temmer, M., Veronig, A., Hanslmeier, A., Otruba, W., \& Messerotti, M. 2001, A\&A, 375, 1049

Tsuneta, S., Acton, L. W., Bruner, M. E., et al. 1991, Sol. Phys., 136, 37

Vaiana, G. S., van Speybroeck, L., Zombeck, M. V., et al. 1977, Space Sci. Instr., 3, 19

Veronig, A., Temmer, M., Hanslmeier, A., Otruba, W., \& Messerotti, M. 2002, A\&A, 382, 1070

Yeung, J., \& Pearce, G. 1990, A\&AS, 82, 543

Wang, H., Gary, D. E., Lim, J., \& Schwartz, R. A. 1994, ApJ, 433, 379 